\title{
AN INTRODUCTION TO THE SYNCHRONIZATION OF CHAOTIC SYSTEMS: COUPLED SKEW TENT MAPS
}

\author{
Martin Hasler \\ Department of Electrical Engineering \\ Swiss Federal Institute of Technology Lausanne \\ 1015 Lausanne, Switzerland \\ and \\ Yuri Maistrenko \\ Institute of Mathematics \\ Academy of Sciences of the Ukraine \\ 3 Tereshchenkivska st. \\ Kiev 252601 Ukraine
}

\begin{abstract}
In this tutorial paper, various phenomena linked to the synchronization of chaotic systems are discussed using the simple example of two coupled skew tent maps. The phenomenon of locally riddled basins of attraction is explained using the Lyapunov exponents transversal to the synchronization manifold. The skew tent maps are coupled in two different ways, leading to quite different global dynamic behavior especially when ideal system is perturbed by parameter mismatch or noise. The linear coupling leads to intermittent desynchronization bursts of large amplitude, whereas for the nonlinear coupling the synchronization error is asymptotically uniformly bounded.
\end{abstract}

\section{Introduction}

The phenomenon of synchronization of two dynamical systems is fundamental in science and has a wealth of applications in technology. While it is natural to associate synchronization with periodic signals, it has in the last decade been realized that chaotic systems can also synchronize [1-4]. This opened the way to envisage engineering applications, the most developed among which is the transmission of information using chaos (for overview articles cf. [5,6]). Recently, various papers in mathematics and physics have been published, which lead to a better understanding of the phenomenon of synchronization [7-12]. The purpose of the present paper is to explain some of their content on the simple example of two coupled skew tent maps, keeping engineering applications in mind.

The iteration of a skew tent map is a well understood chaotic dynamical system for which quantities that are usually difficult to calculate, such as invariant measures and Lyapunov exponents, can be determined explicitly. Two skew tent maps can be coupled in various ways. We use here linear [12] and nonlinear coupling [13]. Formally, the equations of the two systems are very similar, but their synchronization behavior is very different. 
The classification of synchronization behavior used in the mathematical literature is based on the notion of Lyapunov exponents. We first introduce this notion for the iterations of a single skew tent map, and then extend the analysis to two coupled skew tent maps. Whil this analysis gives a good idea of the kind of behavior that can be expected, it is a local analysis in the neighborhood of the synchronization subspace, where the completely synchronized trajectories evolve. Only the properties of the system off the synchronization subspace finally decide which global behavior among the various possibilitites actually takes place. It is usually difficult to prove rigorously properties pertaining to the global behavior.

In our case of nonlinear coupling, we give a proof that all trajectories synchronize when the system has parameters that lie in a certain interval. For the same parameters, we can give bounds on the synchronization error, when there is a parameter mismatch between the two skew tent maps and when noise is intoduced into the equations. Outside of this parameter interval and anywhere for version 1 coupling, we can only give simulation results concerning the global dynamics.

\section{Iterations of the skew-tent map}

We consider the skew tent map f : [0,1] -> [0,1] given by

$$
f(x)=\left\{\begin{array}{ccc}
\frac{x}{a} & \text { if } & 0 \leq x \leq a \\
\frac{1-x}{1-a} & \text { if } & a<x \leq 1
\end{array}\right.
$$

and represented in Fig.1. It is a non-invertible transformation of the unit interval onto itself. It depends on the one parameter a which we suppose to satisfy

$$
0.5 \leq \mathrm{a}<1
$$

The results for $0<\mathrm{a} \leq 0.5$ are completely analogous. For $\mathrm{a}=0.5 \mathrm{f}$ becomes the tent map.

The transformation is continuous and piecewise linear, with the linear regions $[0, a]$ and $[\mathrm{a}, 1]$.

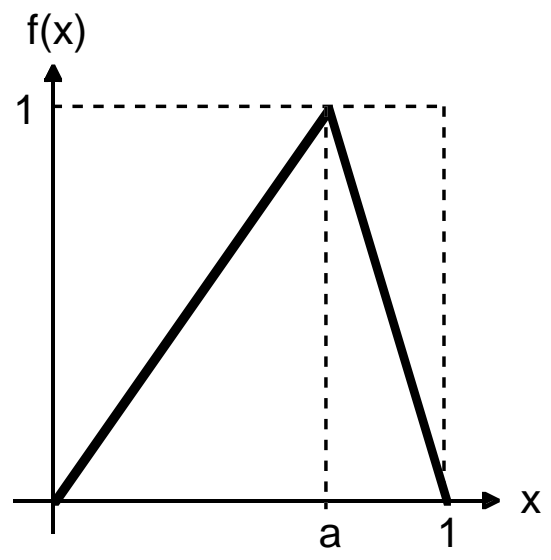


Fig.1. Skew tent map

Note that the slope of the left branch is $1 / \mathrm{a}>1$ and the slope of the right branch $-1 /(1-\mathrm{a})<-1$ and due to (2.2) we have the inequality

$$
1<1 / \mathrm{a} \leq 1 /(1-\mathrm{a})
$$

A typical trajectory $\mathrm{x}(\mathrm{k})$ of the dynamical system obtained by iterating this map, i.e.

$$
\mathrm{x}(\mathrm{k})=\mathrm{f}^{\mathrm{k}}(\mathrm{x}(0))=\mathrm{f}(\mathrm{f}(\ldots \mathrm{f}(\mathrm{x}(0)) \ldots)) \quad \mathrm{k}=0,1,2, \ldots
$$

is shown in Fig.2, for $\mathrm{a}=0.63$.



Fig.2. 300 points of a typical trajectory of the skew tent map system, for $\mathrm{a}=0.63$.

Its waveform is quite irregular and indicates that the system is chaotic. The most important property of a chaotic system is, however, the sensitivity to initial conditions, which will be discussed in the next section. The distribution of the points of a typical orbit of length 1000 $\{\mathrm{x}(\mathrm{k}) \mid \mathrm{k}=0,1, \ldots, 1000\}$ is represented by the histogram of Fig.3. It can be seen that in the course of time, the points of the orbit spread more or less evenly over the unit interval. This property will also be explained in the next section. 


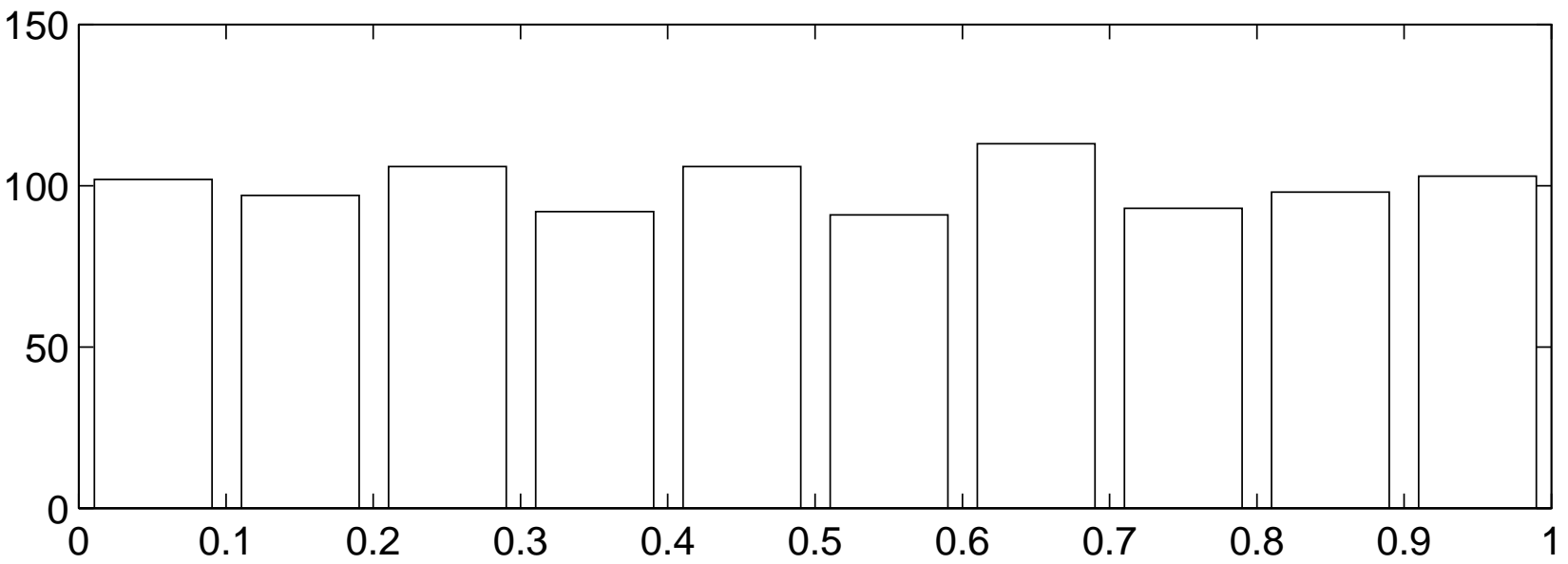

Fig. 3. Histogram of an points of a typical orbit of length 1000

\section{Lyapunov exponents}

The way to measure the sensitivity with respect to initial conditions is to compute the average rate at which nearby trajectories diverge from each other. Consider the trajectories $x(k)$ and $\mathrm{y}(\mathrm{k})$, starting, respectively, from $\mathrm{x}(0)$ and $\mathrm{y}(0)$. If both trajectories are, until time $\mathrm{k}$, always in the same linear region, we can write

$$
|x(j+1)-y(j+1)|=\left|f^{\prime}(x(j))\right||x(j)-y(j)|, \quad \text { for } j=0,1, \ldots, k-1
$$

where $f^{\prime}(x)$ denotes the derivative of $f$ at point $x$. Thus,

$$
|x(k)-y(k)|=\left|f^{\prime}(x(k-1))\right|\left|f^{\prime}(x(k-2))\right| \ldots\left|f^{\prime}(x(0))\right||x(0)-y(0)|
$$

or, equivalently,

$$
|x(k)-y(k)|=e^{\lambda k}|x(0)-y(0)|
$$

where

$$
\lambda=\frac{1}{\mathrm{k}} \sum_{\mathrm{j}=0}^{\mathrm{k}-1} \ln \left|\mathrm{f}^{\prime}(\mathrm{x}(\mathrm{j}))\right|
$$

The interpretation of (6) is that $\lambda$ gives the average rate of divergence (if $\lambda>0$ ), or convergence (if $\lambda<0$ ) of the two trajectories from each other, during the time interval $[0, \mathrm{k}]$. Note that for the tent map (2.1), $\mid \mathrm{f}^{\prime}(\mathrm{x}(\mathrm{j}) \mid$ is either $1 / \mathrm{a}$ or $1 /(1-\mathrm{a})$, but in any case larger than 1 . Thus, $\lambda>0$ and the two trajectories are diverging from each other.

If we follow the two trajectories beyond time $\mathrm{k}$, they eventually will fall into different linear regions, and (3.1) does not hold anymore. However, the closer the initial conditions $\mathrm{x}(0)$ and $\mathrm{y}(0)$ are, the longer in the average it takes, until $\mathrm{x}(\mathrm{k})$ and $\mathrm{y}(\mathrm{k})$ are in different regions. This justifies to interpret the limit of the expression in (3.4) as the average rate of divergence or convergence of nearby trajectories from the given trajectory $\mathrm{x}($.$) , also called the Lyapunov$ exponent of the trajectory. 


\section{Definition 3.1}

The Lyapunov exponent of a trajectory $\mathrm{x}(\mathrm{k})$, given by (2.4), is defined by

$$
\lambda=\lim _{\mathrm{k} \rightarrow \infty}\left[\frac{1}{\mathrm{k}} \sum_{\mathrm{j}=0}^{\mathrm{k}-1}\left|\mathrm{f}^{\prime}(\mathrm{x}(\mathrm{j}))\right|\right]
$$

whenever this expression in the brackets is well defined and the limit exists.

The typical behavior of the expression in the brackets as a function of $\mathrm{k}$ is shown in Fig.4. It can be seen that the convergence is rather slow. From the ergodic theory below it follows that $\lambda$ $=0.6590 \ldots$

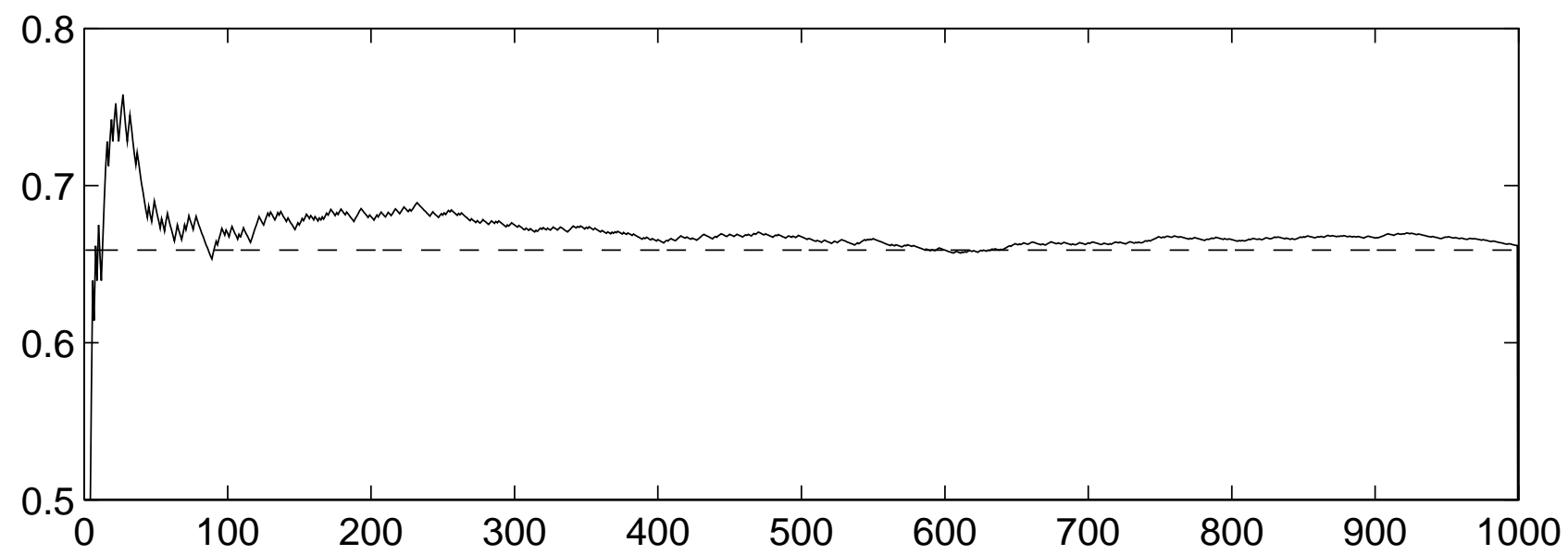

Fig.4. Convergence of the Lyapunov exponent of a typical trajectory, for $\mathrm{a}=0.63$. The asymptotic value should be $\lambda=0.6590 \ldots$ (dotted line)

From (3.5), we can easily deduce a lower and an upper bound for $\lambda$ :

$$
0<-\ln (a) \leq \lambda \leq-\ln (1-a)
$$

For the tent map the lower and upper bound coincide and give $\lambda=\ln (2)=0.6931 \ldots$ For our example $\mathrm{a}=0.63$ the bounds are $0.4620 . . \leq \lambda \leq 0.9943 \ldots$

Note that whenever $x(j)=a$ for some $j, f^{\prime}(x(j))$ is not defined. However, there is only a countable infinity of such trajectories and thus the Lebesgue measure of their initial conditions is zero, even though this set is everywhere dense. Consequently, for (Lebesgue-) almost all initial conditions, the expression in the brackets is well defined and there remains only the question of existence of the limit. Furthermore, we are interested in knowing to which extent $\lambda$ depends on the particular trajectory, once the existence of $\lambda$ is established.

The existence of the limit in (3.5) and its independence, to a certain degree, of the particular trajectory, is linked to the presence of a unique probability density that is invariant under the action of $f$. This is a special case of a natural invariant measure [14].

\section{Definition 3.2}


A probability density $\rho(x)$ on $[0,1]$ is invariant, if for each interval $[c, d] \subset[0,1]$

$$
\int_{c}^{d} \rho(x) d x=\int_{f^{-1}([c, d])} \rho(x) d x
$$

where $\mathrm{f}^{-1}([\mathrm{c}, \mathrm{d}])=\{\mathrm{x} \mid \mathrm{c} \leq \mathrm{f}(\mathrm{x}) \leq \mathrm{d}\}$.

It is not difficult to see that for the tent map (2.1) condition (3.7) amounts to the equation

$$
\rho(x)=a \rho(a x)+(1-a) \rho(1-(1-a) x)
$$

which has as the only solution the constant probability density on [0,1]. It then follows from Birkhoffs ergodic theorem [15] that the asymptotic distribution of the points of almost every trajectory is uniform. This fact is illustrated by Fig.3. The existence and unique value of the Lyapunov exponent also follows from this theorem:

\section{Theorem 3.1.}

Suppose $\mathrm{f}:[0,1]->[0,1]$ is continuously differentiable except for a finite number of points. Let $\rho(\mathrm{x})$ be the unique invariant probability density of the function $\mathrm{f}$. Then for (Lebesgue-) almost all initial conditions, the Lyapunov exponent defined by (3.5) exists and has the value

$$
\lambda^{(\text {nat })}=\int_{0}^{1} \ln \left|f^{\prime}(x)\right| \rho(x) d x
$$

For the skew tent map (2.1) and the constant density, formula (3.9) becomes

$$
\lambda^{(n a t)}=\int_{0}^{\mathrm{a}} \ln \left|\frac{1}{\mathrm{a}}\right| \mathrm{dx}+\int_{\mathrm{a}}^{1-\mathrm{a}} \ln \left|\frac{1}{1-\mathrm{a}}\right| \mathrm{dx}=-\mathrm{a} \ln (\mathrm{a})-(1-\mathrm{a}) \ln (1-\mathrm{a})
$$

In the special case of the tent map $(\mathrm{a}=0.5)$ we find $\lambda=\ln (2)$. For $\mathrm{a}=0.63$ we obtain $\lambda=$ $0.6590 \ldots$, the asymptotic value in Fig.3.

Note that the Lyapunov exponent takes the value given in (3.10) not for all but only for almost all trajectories. For inifinitely many other trajectories, the limit in (3.5) also exists, but its value is different from (3.10). In particular, for any periodic trajectory the limit exist. As a special case, consider the fixed points $x=0$ and $x=1 /(2-a)$. Clearly, for $x=0, \lambda=-\ln (a)$ and for $x=1 /(2-a), \lambda=\ln (1-a)$. Remember that for any trajectory, where the limit exists, its value must satisfy the inequality (3.6). The two fixed points have just the lowest possible and the highest possible Lyapunov exponent.

In numerical experiments, such as the one represented in Fig.4, one obtains the value (3.10) for the Lyapunov exponent. For this reason we call it the natural Lyapunov exponent. of the dynamical system and use the notation $\lambda$ (nat). Why should we worry about the other posssible values for Lyapunov exponents? They are only taken on trajectories that start from a set of initial conditions that has Lebesgue measure 0. It turns out that these Lyapunov exponents play an important role in the synchronization of two coupled tent maps. 


\section{Coupled tent maps and transversal Lyapunov exponent.}

We now couple two skew tent maps in two slightly different ways. This leads to two different two-dimensional maps, $\mathbf{F}$ and $\mathbf{G}$.

\section{Version 1:}

$$
\left(\begin{array}{l}
x(k+1) \\
y(k+1)
\end{array}\right)=\mathbf{F}\left(\begin{array}{l}
x(k) \\
y(k)
\end{array}\right)=\left(\begin{array}{l}
f[x(k)]+\delta(y(k)-x(k)) \\
f[y(k)]+\varepsilon(x(k)-y(k))
\end{array}\right) \quad k=0,1,2, \ldots
$$

Version 2:

$$
\left(\begin{array}{l}
x(k+1) \\
y(k+1)
\end{array}\right)=\mathbf{G}\left(\begin{array}{l}
x(k) \\
y(k)
\end{array}\right)=\left(\begin{array}{l}
f[x(k)+\delta(y(k)-x(k))] \\
f[y(k)+\varepsilon(x(k)-y(k))]
\end{array}\right) \quad k=0,1,2, \ldots
$$

The two coupling parameters $\delta$ and $\varepsilon$ can take any value. As it will turn out, the qualitative behavior of the system depends essentially on $\delta+\varepsilon$. If we set $\delta=0, \mathrm{x}(\mathrm{k})$ is not influenced by $\mathrm{y}(\mathrm{k})$, but unless at the same time $\varepsilon=0, \mathrm{x}(\mathrm{k})$ influences $\mathrm{y}(\mathrm{k})$. We call this coupling a masterslave configuration.

Note that the map $\mathbf{F}$ does not leave the square $[0,1]^{2}$ invariant, unless both $\delta$ and $\varepsilon$ are zero, whereas $\mathbf{G}$ leaves the square invariant when both $\delta \geq 0$ and $\varepsilon \geq 0$. In fact, in order to obtain in all cases a well-defined $\mathbf{F}$ and $\mathbf{G}$ as transformations of $\mathrm{R}^{2}$, we have to extend the skew tent map to the whole of $\mathrm{R}$ by omitting the restrictions $0 \leq \mathrm{x}$ and $\mathrm{x} \leq 1$ in (2.1). In any case, both $\mathbf{F}$ and G leave the staight line $\mathrm{x}=\mathrm{y}$ of the plane, as well as its part in $[0,1]^{2}$, the segment

$$
\mathrm{S}=\{(\mathrm{x}, \mathrm{y}) \mid 0 \leq \mathrm{x}=\mathrm{y} \leq 1\}
$$

invariant. The straight line $\mathrm{x}=\mathrm{y}$ is the subspace, where the two dynamical systems $\mathrm{x}(\mathrm{k}+1)=$ $\mathrm{f}(\mathrm{x}(\mathrm{k}))$ and $\mathrm{y}(\mathrm{k}+1)=\mathrm{f}(\mathrm{y}(\mathrm{k}))$ are synchronized. Accordingly, we say that any trajectory that starts elsewhere in the plane, but converges to the subspace $\mathrm{x}=\mathrm{y}$, synchronizes. In Fig. 5, a synchronizing orbit of the system (4.2) is represented, which is attracted to the segment $\mathrm{S}$ while remaining chaotic. Indeed, within $\mathrm{S}$, the dynamics is one-dimensional, and given by the iterations of the map $f$. 


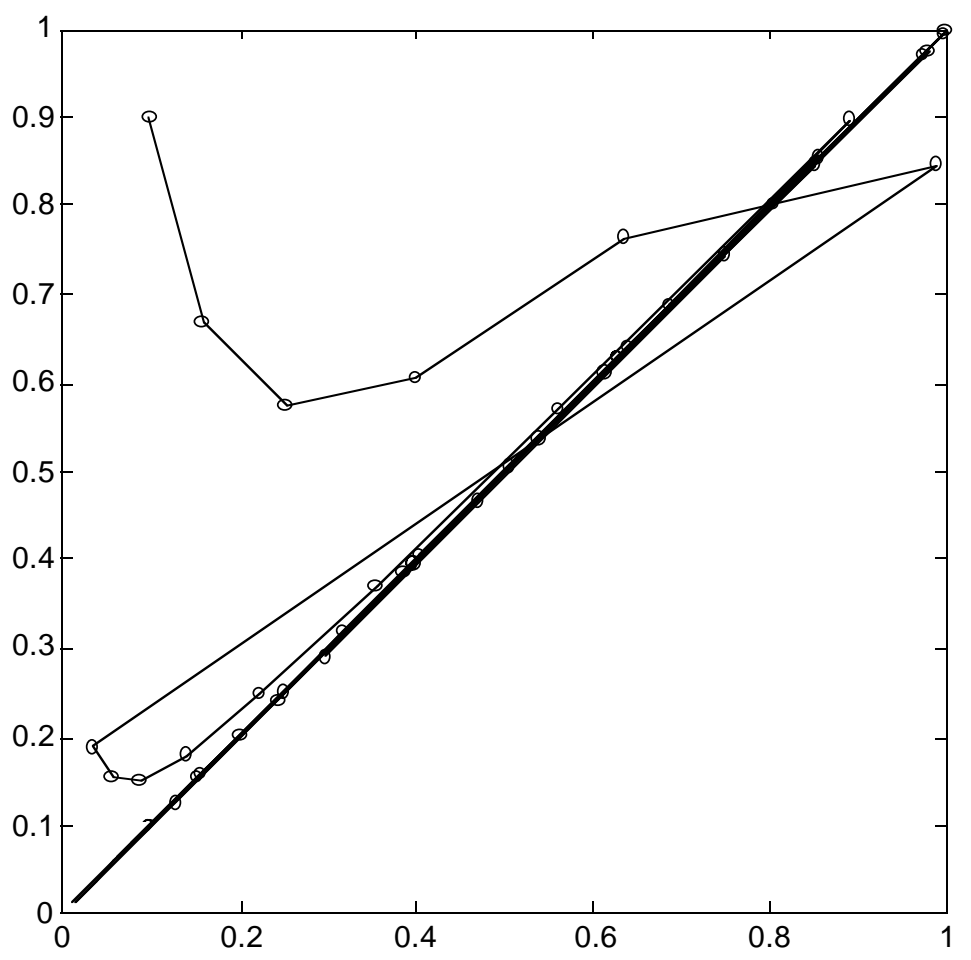

Fig. 5. Synchronizing trajectory of the system (4.2) with $\mathrm{a}=0.63, \delta=0, \varepsilon=0.6$ and $(\mathrm{x}(0), \mathrm{y}(0))=(0.1,0.9)$

Note, however, that any trajectory that starts on the straight line beyond $\mathrm{S}$ diverges to $(-\infty$, $-\infty)$. Thus, a trajectory starting outside of $\mathrm{x}=\mathrm{y}$ may diverge to infinity while approaching $\mathrm{x}=$ y. Since this is not desirable in applications, we exclude divergence to infinity in the notion of synchronization.

\section{Definition 4.1.}

A trajectory of the dynamical system defined by (4.1) or (4.2) synchronizes if it is bounded and

$$
|\mathrm{x}(\mathrm{k})-\mathrm{y}(\mathrm{k})| \underset{\mathrm{k} \rightarrow+\infty}{\longrightarrow} 0
$$

This means that asymptotically the waveforms $x(k)$ and $y(k)$ are identical, even if they are of chaotic nature, as in Fig.2. In Fig.6 the two waveforms are represented for the trajectory of Fig.5. 

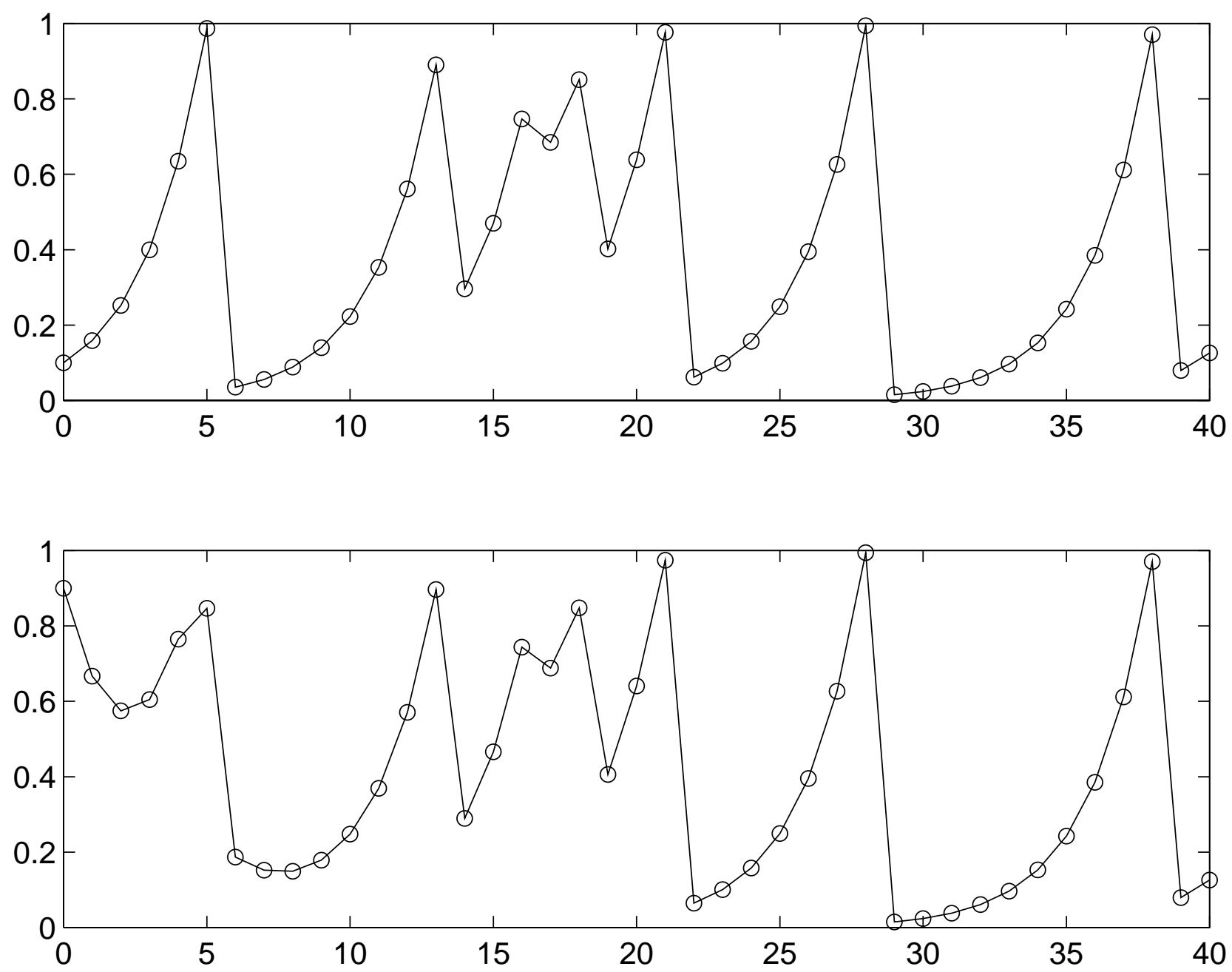

Fig. 6. Waveforms $x(k)$ and $y(k)$ for the trajectory of Fig.5

The question now arises, for which initial conditions in $[0,1]^{2}$ synchronization takes place. Since boundedness is required in definition 4.1, this is equivalent to asking for which initial conditions the trajectory converges to S. If synchronization is desired, as in the transmission of information on a chaotic carrier signal $[5,6]$, then we should have synchronization for all trajectories starting in $[0,1]^{2}$. While this behavior indeed is possible, at least for version 2 , other, more complicated phenomena may appear, as we shall see.

The purpose of this paper is to describe and explain these phenomena using the simple example of coupled skew tent maps, and not to optimize the parameters for the synchronization of the example system. Otherwise, we could simply take version 2 coupling with $\delta=0$ and $\varepsilon=$ 1 , for which perfect synchronization is achieved in one iteration.

For applications, we still could be satisfied, if for any initial condition in a neighborhood of $S$ synchronization takes place. In order to check this local stability of the synchronization subspace, we have to investigate, whether a trajectory that starts slightly off $S$ converges to a trajectory in S or whether it diverges from S. Again, we consider the Lyapunov exponents as a measure of the speed of mutual convergence or divergence of trajectories. In $\mathrm{N}$ dimensions they are defined as follows. 


\section{Definition 4.2.}

The Lyapunov exponents of a trajectory $\mathbf{x}(\mathrm{k})$ obtained by iteration of a map $\mathbf{F}: \mathrm{R}^{\mathrm{N}} \rightarrow>\mathrm{R}^{\mathrm{N}}$ are defined to be the eigenvalues of the matrix

$$
\Lambda=\lim _{\mathrm{k} \rightarrow \infty} \frac{1}{2 \mathrm{k}} \ln \left[\mathbf{D}_{\mathrm{k}}^{\mathrm{T}} \mathbf{D}_{\mathrm{k}}\right]
$$

whenever $\mathbf{D}_{\mathrm{k}}$ is well-defined and the limit exists. Here $\mathbf{D}^{\mathrm{T}}$ denotes the transpose of $\mathbf{D}$ and

$$
\mathbf{D}_{\mathrm{k}}=\frac{\partial \mathbf{F}}{\partial \mathbf{x}}(\mathbf{x}(\mathrm{k}-1)) \frac{\partial \mathbf{F}}{\partial \mathbf{x}}(\mathbf{x}(\mathrm{k}-2)) \ldots \frac{\partial \mathbf{F}}{\partial \mathbf{x}}(\mathbf{x}(0))
$$

where $\frac{\partial \mathbf{F}}{\partial \mathbf{x}}(\mathbf{x}(\mathrm{j}))$ is the Jacobian matrix of $\mathbf{F}$ at the point $\mathbf{x}(\mathrm{j})$.

For dimension $\mathrm{N}=1$, there is only one Lyapunov exponent and the expression (4.5) reduces to (3.5). In general, for $\mathrm{N}>1$, Birkhoff's ergodic theorem cannot be used anymore to prove the existence of the Lyapunov exponents. Instead, Oseledec's non-commutative ergodic theorem [16] can be applied, which does give the existence of the Lyapunov exponents and their independence from the particular trajectory for almost all initial conditions. Contrary to Birkhoff's theorem, however, Oseledec's theorem does not give an explicit expression of the Lyapunov exponents in terms of an invariant measure.

In our case, fortunately, the eigenvectors of the Jacobian matrix are independent of the particular point on the synchronization subspace $\mathrm{x}=\mathrm{y}$. We first discuss this for the system of version 1. The Jacobian matrix becomes in this case

$$
\begin{aligned}
& \mathbf{D}\left(\left(\begin{array}{l}
\mathrm{x} \\
\mathrm{x}
\end{array}\right)\right)=\left(\begin{array}{cc}
\mathrm{c}-\delta & \delta \\
\varepsilon & \mathrm{c}-\varepsilon
\end{array}\right) \\
& \text { where } \mathrm{c}=\left\{\begin{array}{c}
\frac{1}{\mathrm{a}} \text { if } \mathrm{x} \leq \mathrm{a} \\
-\frac{1}{1-\mathrm{a}} \text { if } \mathrm{a}<\mathrm{x}
\end{array}\right.
\end{aligned}
$$

Its eigenvectors are

$$
\mathbf{e}_{1}=\left(\begin{array}{l}
1 \\
1
\end{array}\right), \quad \mathbf{e}_{2}=\left(\begin{array}{c}
\delta \\
-\varepsilon
\end{array}\right)
$$

and the corresponding eigenvalues

$$
\mu_{1}(\mathrm{x})=\left\{\begin{array}{l}
\frac{1}{\mathrm{a}} \text { if } \mathrm{x} \leq \mathrm{a} \\
-\frac{1}{1-\mathrm{a}} \text { if } \mathrm{a}<\mathrm{x}
\end{array} \quad \mu_{2}(\mathrm{x})=\left\{\begin{array}{c}
\frac{1}{\mathrm{a}}-\delta-\varepsilon \text { if } \mathrm{x} \leq \mathrm{a} \\
-\frac{1}{1-\mathrm{a}}-\delta-\varepsilon \text { if } \mathrm{a}<\mathrm{x}
\end{array}\right.\right.
$$

Since the eigenvectors do not depend on $\mathrm{x}$, they are also eigenvectors of $\mathbf{D}_{\mathrm{k}}$. The two eigen values of $\mathbf{D}_{\mathrm{k}}$ are thus 


$$
\mu_{\mathrm{i}}=\mu_{\mathrm{i}}(\mathrm{x}(\mathrm{k}-1)) \ldots \mu_{\mathrm{i}}(\mathrm{x}(1)) \mu_{\mathrm{i}}(\mathrm{x}(0)), \quad \mathrm{i}=1,2
$$

and the two Lyapunov exponents

$$
\lambda_{\mathrm{i}}=\lim _{\mathrm{k} \rightarrow \infty} \frac{1}{\mathrm{k}} \sum_{\mathrm{j}=0}^{\mathrm{k}-1} \ln \left|\mu_{\mathrm{i}}(\mathrm{x}(\mathrm{j}))\right|
$$

The first eigenvector lies in the subspace $\mathrm{x}=\mathrm{y}$. Thus, the first Lyapunov exponent corresponds to the motion in the subspace $S$ and this motion is given by the one-dimensional dynamical system (2.4). For this reason we use the notation $\lambda_{\|}$instead of $\lambda_{1}$.

The second eigenvector is transversal to the subspace $\mathrm{x}=\mathrm{y}$. The corresponding Lyapunov exponent is called transversal Lyapunov exponent and denotedby $\lambda_{\perp}$ instead of $\lambda_{2}$.

Using the one-dimensional invariant probability density $\rho$ on $S$, the value of $\lambda_{i}$ for almost all trajectories in $\mathrm{S}$ can again be computed explicity by applying Birkhoff's ergodic theorem:

$$
\lambda_{i}^{(\text {nat })}=\int_{0}^{1} \ln \left|\mu_{i}(x)\right| \rho(x) d x
$$

For the natural parallel Lyapunov exponent $\lambda_{\|}{ }^{(\text {nat })}$ we obtain the value (3.10) and for the natural transversal Lyapunov exponent, computed by (4.12), the value

$$
\begin{aligned}
& \lambda_{\perp}^{(\text {nat })}=\mathrm{a} \ln \left|\frac{1}{\mathrm{a}}-\mathrm{d}\right|+(1-\mathrm{a}) \ln \left|-\frac{1}{1-\mathrm{a}}-\mathrm{d}\right| \\
& \text { where } \mathrm{d}=\delta+\varepsilon
\end{aligned}
$$

If $\lambda_{\perp}$ (nat) is negative, the trajectories starting close to $S$ are in the mean attracted to $S$. We expect this to be the condition for synchronization. As we shall see, it is indeed a necessary condition for synchronization, but it is not sufficient.

Let us discuss for which coupling constants $\delta$ and $\varepsilon \lambda_{\perp}$ (nat) is negative. In fact, the only parameter that matters is $d=\delta+\varepsilon$. Consider first the special case of the standard tent map ( $a=$ 1/2). The condition becomes

$$
0>\frac{1}{2} \ln |2-\mathrm{d}|+\frac{1}{2} \ln |2+\mathrm{d}|=\frac{1}{2} \ln \left|\left(4-\mathrm{d}^{2}\right)\right|
$$

or, equivalently,

$$
\left|4-\mathrm{d}^{2}\right|<1
$$

This condition is satisfied in the two intervals $-\sqrt{5}<d<-\sqrt{3}$ and $\sqrt{5}<d<\sqrt{ } 3$ (Fig.7).

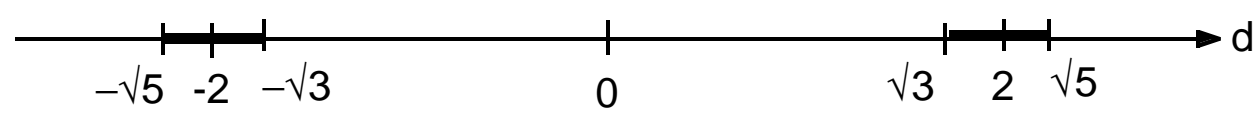


Fig. 7. Intervals with negative natural transversal Lyapunov exponent for the standard tent map in the case of coupling according to version 1.

In general, the following property holds:

\section{Property 4.1.}

Two skew tent maps with identical parameter value $0<\mathrm{a}<1$ and version 1 coupling have a negative natural transversal Lyapunov exponent whenever the coupling parameter $\mathrm{d}=$ $\delta+\varepsilon$ belongs to one of the two intervals $\left(d_{1}{ }^{(-)}, d_{2}{ }^{(-)}\right),\left(d_{1}{ }^{(+)}, d_{2}{ }^{(+)}\right)$where the end points of the intervals are the roots of the transcendental equation

$$
\lambda_{\perp}^{(\text {nat })}=a \ln \left|\frac{1}{a}-d\right|+(1-a) \ln \left|-\frac{1}{1-a}-d\right|=0
$$

They satisfy (Fig.8)

$$
-\infty<\mathrm{d}_{1}^{(-)}<-\frac{1}{1-\mathrm{a}}<\mathrm{d}_{2}^{(-)}<0<\mathrm{d}_{1}^{(+)}<\frac{1}{\mathrm{a}}<\mathrm{d}_{2}^{(+)}<+\infty
$$

Moreover, $\lambda_{\perp}$ as a function of $\mathrm{d}$ has the following properties:

a) $\lambda_{\perp}$ (nat) $\rightarrow>-\infty$ as $d->-1 /(1-a)$ and $d->1 / a$

b) $\lambda_{\perp}$ (nat) $->+\infty$ as $d-> \pm \infty$

c) $\lambda_{\perp}{ }_{\perp}^{(n a t)}(d)$ has exactly one finite extremum, namely a maximum at

$$
\mathrm{d}^{*}=\frac{1-2 \mathrm{a}}{\mathrm{a}(1-\mathrm{a})} \in\left(-\frac{1}{1-\mathrm{a}}, \frac{1}{\mathrm{a}}\right)
$$

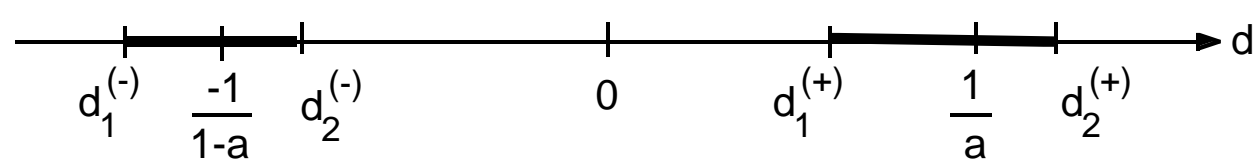

Fig.8. Intervals with negative natural transversal Lyapunov exponent for the skew tent map in case of coupling according to version 1.

In Fig.9 $\lambda_{\perp}{ }^{\text {(nat) }}$ is represented as a function of $d$. 


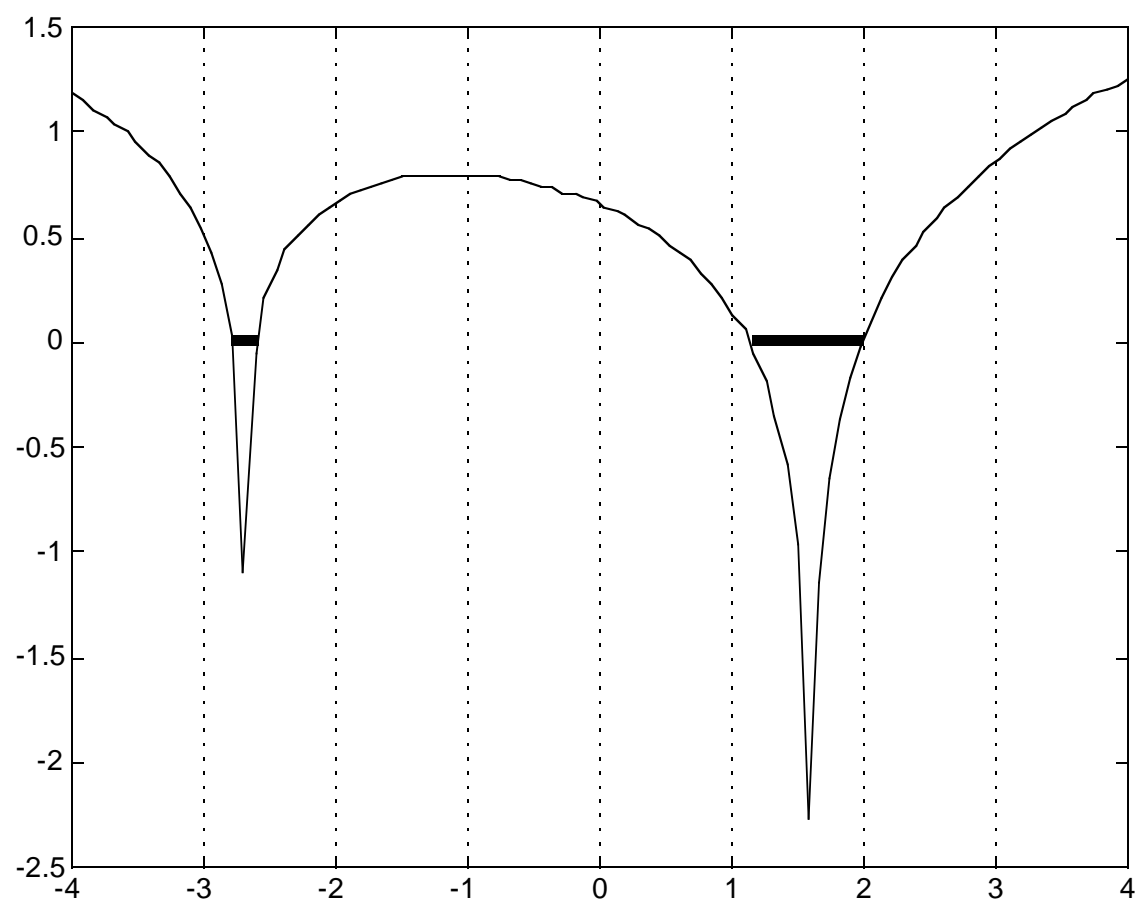

Fig.9. The natural transversal Lyapunov exponent as a function of the coupling parameter $d=\delta+\varepsilon$, for the coupling according to version 1 . As can be guessed from this figure, at two values of $d$, the Lyapunov exponent is $-\infty$. In the two intervals indicated in bold, the Lyapunov exponent is negative.

For the system of version 2 the Jacobian matrix for $\mathrm{x}=\mathrm{y}$ is

$$
\begin{aligned}
& \mathbf{D}\left(\left(\begin{array}{l}
\mathrm{x} \\
\mathrm{x}
\end{array}\right)\right)=\mathrm{c}\left(\begin{array}{cc}
1-\delta & \delta \\
\varepsilon & 1-\varepsilon
\end{array}\right) \\
& \text { where again } \mathrm{c}=\left\{\begin{array}{c}
\frac{1}{\mathrm{a}} \text { if } 0 \leq \mathrm{x} \leq \mathrm{a} \\
-\frac{1}{1-\mathrm{a}} \text { if } \mathrm{a}<\mathrm{x} \leq 1
\end{array}\right.
\end{aligned}
$$

The eigenvectors as well as the eigenvalue $\mu_{1}(\mathrm{x})$ are the same as in version 1 (cf. (4.8), (4.9)), and the eigenvalue $\mu_{2}(\mathrm{x})$ is given by

$$
\mu_{2}(x)=\left\{\begin{array}{cl}
\frac{1}{a}(1-\delta-\varepsilon) & \text { if } 0 \leq x \leq a \\
-\frac{1}{1-a}(1-\delta-\varepsilon) & \text { if } a<x \leq 1
\end{array}\right.
$$

This leads to the natural transversal Lyapunov exponent

$$
\begin{aligned}
& \lambda_{\perp}^{\text {(nat) }}=-\mathrm{a} \ln (\mathrm{a})-(1-\mathrm{a}) \ln (1-\mathrm{a})+\ln |1-\mathrm{d}| \\
& \text { where again } \mathrm{d}=\delta+\varepsilon
\end{aligned}
$$


When $|1-\mathrm{d}|$ is sufficiently small, the natural transversal Lyapunov exponent is negative.

\section{Property 4.2.}

Two skew tent maps with identical parameter value $0<a<1$ and version 2 coupling has a negative natural transversal Lyapunov exponent whenever the coupling parameter $d=\delta+\varepsilon$ belongs to the interval $[1-\Delta, 1+\Delta]$, where $\Delta$ is given by

$$
\ln (\Delta)=\operatorname{aln}(\mathrm{a})+(1-\mathrm{a}) \ln (1-\mathrm{a})
$$

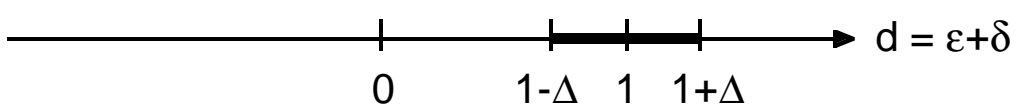

Fig.10. Interval with negative natural transversal Lyapunov exponent for the skew tent map with coupling according to version 2

\section{The notion of attractor and synchronization for two coupled identical skew tent maps}

We now investigate more closely the synchronization of trajectories that start close to the segment $\mathrm{S}$ given by (4.3), when the natural transversal Lyapunov exponent is negative. In the terminology of dynamical system theory, we study in which sense $\mathrm{S}$ is an attractor. The various notions of attractors involve two kinds of properties: Some property that it attracts closeby trajectories, and some property of minimality, i.e. that it cannot be decomposed into smaller attractors. We shall concentrate on the first property, since $S$ has everywhere dense trajectories. Thus, the definitions given here should be completed by some minimality condition in order to be generally valid.

Together with an attractor, one considers its basin of attraction, or simply basin, which is the set of all initial conditions from which the trajectories converge to the attractor. For our attractor $\mathrm{S}$, it is the set of all point from which synchronizing trajectories start. Usually, basins of attraction are open sets with a simple geometric structure. In the context of synchronization of chaos, riddled basins have been observed [7-12], i.e. basins that nowhere contain any open balls, even not very tiny ones, but nevertheless have positive Lebesgue measure. As we shall see, for the systems we consider the basins do not seem to be riddled, but only locally riddled (cf. theorem 5.3).

The property that the natural transversal Lyapunov exponent is negative means that almost all synchronized trajectories, i.e. almost all trajectories that are confined to $\mathrm{S}$ are transversally attracting. This implies that $\mathrm{S}$ is an attractor in the weak Milnor sense.

\section{Theorem 5.1.}


If the natural transversal Lyapunov exponent is negative, the set $\mathrm{S}$ is an attractor in the weak Milnor sense, i.e. a set of trajectories whose initial conditions have positive measure $\mathrm{m}_{\eta}$ in any neighborhood $U_{\eta}(S)$ of $S$ converge to $S$, where

$$
\mathrm{U}_{\eta}(\mathrm{S})=\{(\mathrm{x}, \mathrm{y}) \mid \forall \mathrm{v} \text { with } 0 \leq \mathrm{v} \leq 1 \|(\mathrm{x}-\mathrm{v}, \mathrm{y}-\mathrm{v} \|<\eta\}
$$

In other words, in any neighborhood of $S$ there is a set of positive measure $m_{\eta}$ of initial conditions of synchronizing trajectories. Moreover,

$$
m_{\eta} \rightarrow 1 \text { as } \eta \rightarrow 0
$$

\section{Proof:}

See $[7,11]$.

The property that $\mathrm{S}$ is an attractor in the weak Milnor sense is indeed weak and allows still for much exotic behavior of the trajectories, which is undesirable in applications. Good synchronization, at least locally, takes place when $\mathrm{S}$ is an asymptotically stable attractor i.e.

- when all trajectories in a neighborhood $U_{\eta}$ converge to $S$

- and when $\mathrm{S}$ is Lyapunov stable.

In somewhat inaccurate but intuitive terms, the second condition means that once a trajectory is sufficiently close to $\mathrm{S}$, it will subsequently not go far away from $\mathrm{S}$. In practical applications, both conditions are important.

Clearly, these conditions are not satisfied because the trajectories starting at $(-\eta / 2,-\eta / 2)$ diverge to $(-\infty,-\infty)$. This could be avoided by modifying slightly the function $\mathrm{f}$. Apart from this problem there may be trajectories within $S$ that are transversally repelling, i.e. whose transversal Lyapunov exponent is positive. We first specify the coupling constants for which this is the case.

\section{Property 5.2.}

a) The system of two skew tent maps coupled according to version 1 (cf. eq. (4.1)) has trajectories in the synchronization segment $S$ (cf. eq. (4.3)) with positive transversal Lyapunov exponent for any value of the coupling constants $\delta$ and $\varepsilon$. The initial conditions of these trajectories are dense in $\mathrm{S}$.

b) In system of two skew tent maps coupled according to version 2 (cf. eq. (4.2)) with

$$
1-\Delta_{0}<\mathrm{d}=\varepsilon+\delta<1+\Delta_{0} \text {, where } \Delta_{0}=\min \{\mathrm{a}, 1-\mathrm{a}\}
$$

all synchronized trajectories are transversally attracting, i.e. all trajectories in $\mathrm{S}$ have a negative transversal Lyapunov exponent (Fig.11). For any d lying outside of the closure of the interval (5.3), there is a dense set of initial conditions that generate trajectories with positive transversal Lyapunov exponents. 


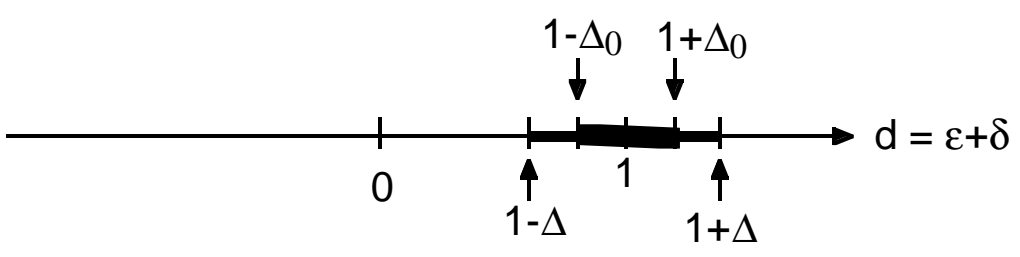

Fig. 11. Skew tent maps coupled according to version 2. Interval for the parameter d (strongly bold) where all trajectories in $\mathrm{S}$ have a negative transversal Lyapunov exponent, within the interval (bold), where the natural transversal Lyapunov exponent is negative.

\section{Proof:}

a) The transversal Lyapunov exponents for the constant trajectories at the two fixed points $(0,0)$ and $\left(\frac{1}{2-\mathrm{a}}, \frac{1}{2-\mathrm{a}}\right)$ are, according to (4.11) and (4.9)

$$
\lambda_{\perp}=\ln \left|\frac{1}{\mathrm{a}}-\mathrm{d}\right|
$$

and

$$
\lambda_{\perp}=\ln \left|-\frac{1}{1-\mathrm{a}}-\mathrm{d}\right|
$$

respectively. Expression (5.4) can only be negative for $\mathrm{d}>0$ and expression (5.5) for $\mathrm{d}<$ 0 . Thus, for any $d$, at least one of the two transversal Lyapunov exponents is positive. Furthermore, the inverse images of the two fixed points under $\mathbf{F}$ are dense in S. All trajectories starting at such points are eventually constant and have therefore the Lyapunov exponents of the corresponding fixed points. Therefore, for any d, a dense set of initial conditions in $\mathrm{S}$ leads to trajectories with positive transversal Lyapunov exponents.

b) According to (4.11) and (4.20), the transversal Lyapunov exponent of any trajectory in $S$ is bounded by

$$
\lambda_{\perp} \leq \max \{-\ln (\mathrm{a}),-\ln (1-\mathrm{a})\}-\ln (1-\mathrm{d})
$$

If

$$
|1-\mathrm{d}|<\min \{\mathrm{a}, 1-\mathrm{a}\}
$$

the RHS od (5.6) is negative and therefore the transversal Lyapunov exponent of any trajectory in $\mathrm{S}$ is negative. On the other hand, the bound (5.6) is reached for the constant trajectories sitting at one of the two fixed points. If the strict inequality opposite to (5.7) holds, the corresponding transversal Lyapunov exponent is positive. In this case, again the preimages of the fixed point provides the dense set of initial conditions in $S$ that lead to trajectories with positive Lyapunov exponents.

We now discuss the synchronization properties for the different cases. 


\section{Theorem 5.3.}

a) Version 1 coupling: If the natural transversal Lyapunov exponent is negative, $S$ is aa attractor with a locally riddled basin, i.e. there is a neighborhood $\mathrm{U}$ of $\mathrm{S}$ such that in any neighborhood $\mathrm{V}$ of any point in $\mathrm{S}$, there is a set of points in $\mathrm{V} \cap \mathrm{U}$ of positive measure which leave $U$ in a finite time (Fig.12).

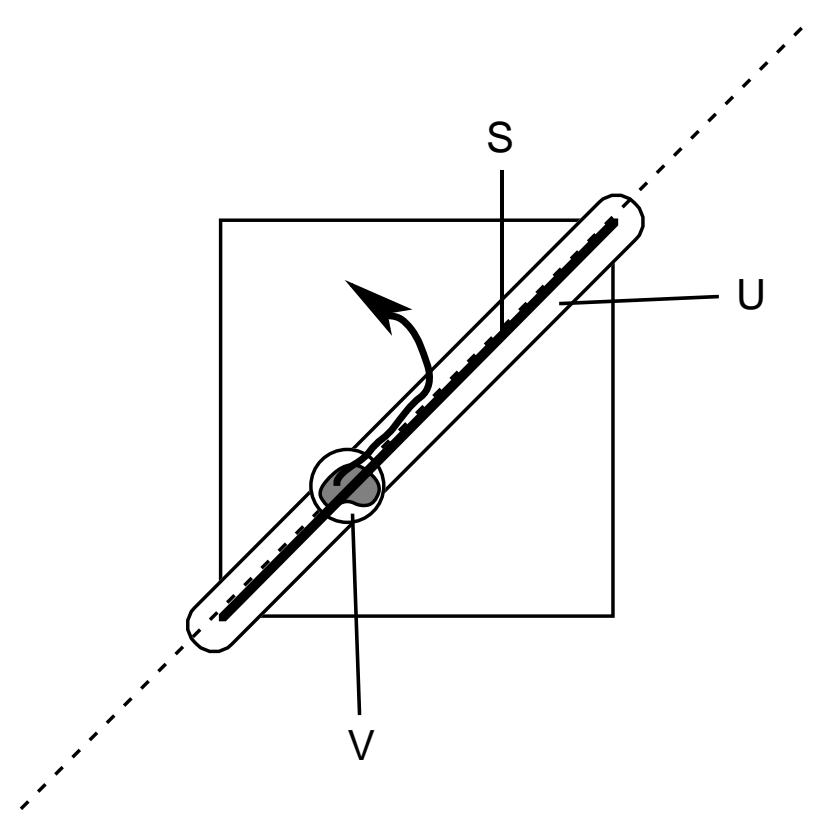

Fig.12. Locally riddled attractor

b) Version 2 coupling: If inequality (5.3) is satisfied and if $\varepsilon, \delta \geq 0$ and the dynamical system is restricted to the square $[0,1]^{2}, \mathrm{~S}$ is an globally asymptotically stable attractor. In fact, all trajectories synchronize with an exponential synchronization speed:

$$
|\mathrm{x}(\mathrm{k})-\mathrm{y}(\mathrm{k})| \leq \gamma^{\mathrm{k}}|\mathrm{x}(0)-\mathrm{y}(0)|
$$

where

$$
\gamma=\max \left\{\frac{|1-\mathrm{d}|}{\mathrm{a}}, \frac{|1-\mathrm{d}|}{1-\mathrm{a}}\right\}
$$

If the inequality opposite to (5.3) holds, but the natural transversal Lyapunov exponent is still negative, $\mathrm{S}$ is an attractor with a locally riddled basin (Fig.12).

\section{Proof:}

For a rigorous proof of the locally riddled basin property, cf. [7,11]. Here, we only give an intuitive explanation. If the natural transversal Lyapunov exponent is negative, but at the same time there is a periodic trajectory in $\mathrm{S}$ with a positive transversal Lyapunov exponent, there is neighborhood $\mathrm{U}$ of $\mathrm{S}$ and a subset $\mathrm{V}$ of $\mathrm{U}$ of "tongues" emanating from the points of this trajectory such that any trajectory close to $\mathrm{S}$ (but not in $\mathrm{S}$ ) that falls into $\mathrm{V}$ can leave $\mathrm{V}$ only through the boundary of U (Fig.13). All preimages of periodic trajectories with positive 
transversal Lyapunov exponent also carry such "ejecting tongues". Since these preimages are dense in $\mathrm{S}$, one can easily imagine that starting from any point near $\mathrm{S}$, there is a non-zero probability that a trajectory is pushed away from $\mathrm{S}$ through one of the tongues.

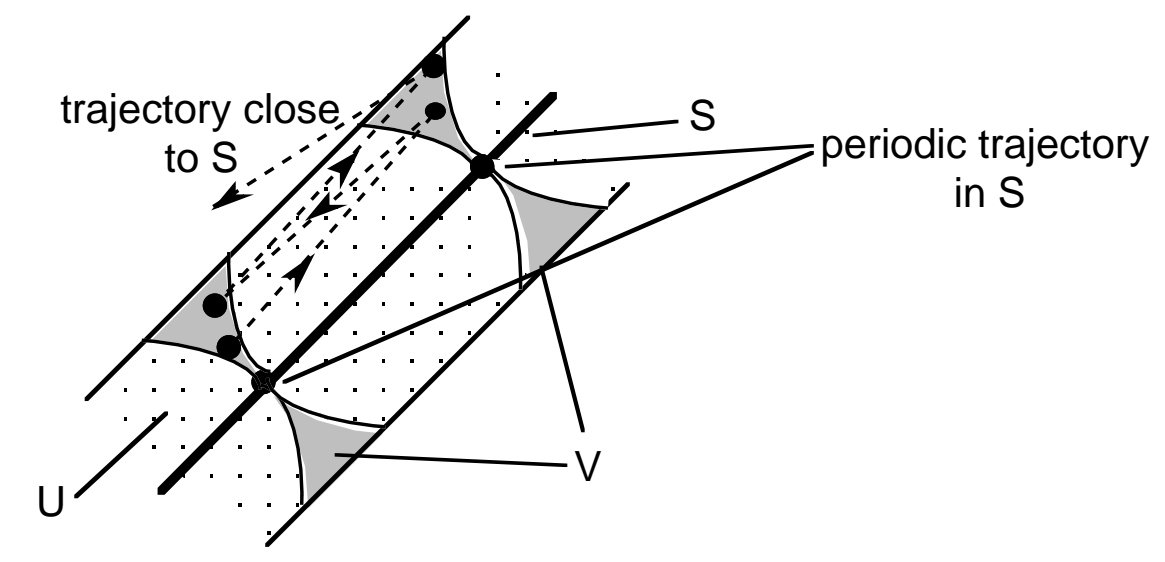

Fig.13. "Tongues" emanating from a periodic trajectory in $\mathrm{S}$ which eject a trajectory close to $\mathrm{S}$ away from $\mathrm{S}$

To prove (5.8):

$$
\begin{aligned}
|\mathrm{x}(\mathrm{k})-\mathrm{y}(\mathrm{k})| & =|\mathrm{f}[(1-\delta) \mathrm{x}(\mathrm{k}-1)+\delta \mathrm{y}(\mathrm{k}-1)]-\mathrm{f}[(1-\varepsilon) \mathrm{x}(\mathrm{k}-1)+\mathrm{y}(\mathrm{k}-1)]| \\
& \leq \max \left\{\frac{1}{\mathrm{a}}, \frac{1}{1-\mathrm{a}}\right\}(1-\delta-\varepsilon)(\mathrm{x}(\mathrm{k}-1)-\mathrm{y}(\mathrm{k}-1)) \mid \\
& =\max \left\{\frac{|1-\mathrm{d}|}{\mathrm{a}}, \frac{|1-\mathrm{d}|}{1-\mathrm{a}}\right\}|\mathrm{x}(\mathrm{k}-1)-\mathrm{y}(\mathrm{k}-1)|
\end{aligned}
$$

which implies (5.8).

Thus, when the natural transversal Lyapunov exponent is negative, two cases have to be distinguished:

- the transversal Lyapunov exponent of all trajectories in $\mathrm{S}$ is negative and $\mathrm{S}$ is asymptotically stable. In our case it is even gloabally asymptotically stable, i.e.all trajectories in $[0,1]^{2}$ synchronize.

- some trajectories have a positive transversal Lyapunov exponent and $\mathrm{S}$ has a locally riddled basin of attraction. This means that some trajectories starting close to $S$ remain close to $S$ and eventually converge to $S$ whereas others go farther away from $S$. In more technical terms: $S$ is not Lyapunov stable. What happens to such trajectories afterwards is an open question which cannot be decided on the basis of local properties of the dynamics around S. At this point the global dynamics come into play [17].

In the second case, the global behavior of may be:

- convergence to $\mathrm{S}$. In this case the synchronization error shows a few bursts and then converges to zero 
- converge to some other attractor. In this case there is no synchronization at all, except that there maybe be some time intervals initially, when the synchronization error is small.

- does not converge. In this case the trajectory may wander around anywhere in the plane, but most ltypically, it will alternate intervals of approximate synchronization with desynchronization bursts forever.

If we consider all possible trajectories together, some very different behavior may take place:

- the "best" synchronization in the presence of a locally riddled basin: all trajectories starting close to $\mathrm{S}$ synchronize. Nevertheless, $\mathrm{S}$ is not Lyapunov stable, and most trajectories will show a few of desynchronization bursts.

- a riddled basin of attraction: there is a set of positive measure of starting points for trajectories which synchronize, but arbitrily close to any such point there are other initial condition whose trajectories converge to another attractor, or dont converge at all.

We have performed a number of simulations of two coupled skew tent maps with $a=0.63$ in order to obtain an idea of the global dynamics. For version 1 coupling, the natural transversal Lyapunov exponent is negative in the intervals for $d=\delta+\varepsilon(1.133 . ., 1.990 .$.$) and$ $(-2.783 . .,-2.615)$. All computed trajectories converged to $S$ as long as the natural transversal Lyapunov exponent was negative. For the master-slave configuration $\delta=0, \varepsilon=1.135$ the natural transversal Lyapunov exponent is only slightly negative, namely $\lambda_{\perp}{ }^{\text {(nat })}=-0.0022 \ldots$. In Fig.14, a trajectory of this system is represented. Note the desynchronization burst after a long period of apparent synchronization. Despite the value of the Lyapunov exponent very close to zero, after 391 steps, perfect synchronization was achieved which excludes further desynchronization bursts. Thus, the synchronization behavior appears to be the best possible in the presence of positive transversal Lyapunov exponents and thus a locally riddled basin of attraction for $\mathrm{S}$.

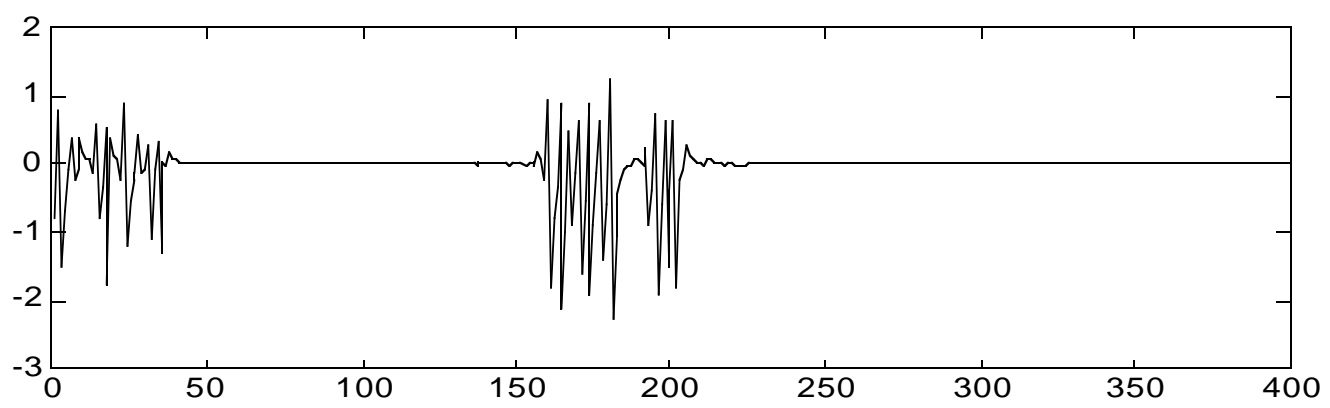

Fig.14. Synchronization error of a trajectory of two skew tent maps coupled according to version 1 in master-slave configuration, with $\delta=0$ and $\varepsilon=1.135$, starting from $\mathrm{x}(1)=$ $0.9, \mathrm{y}(1)=0.1$.

For version 2 coupling of skew tent maps with parameter a $=0.63$, the natural transversal Lyapunov exponent is negative in the interval $(0.4826 . ., 1.5174 .$.$) of the coupling$ parameter $d=\delta+\varepsilon$. In the interval $(0.63,1.37)$ all transversal Lyapunov exponents are negative. Again, the computed trajectories starting outside of $S$ all synchronized exactly even when certain trajectories in $\mathrm{S}$ have a positive transversal Lyapunov exponent. We present a 
similar case as for the version 1 coupling, namely a master-slave coupling $\delta=0, \varepsilon=0.484$, which produces a natural transversal Lyapunov exponenet of $-0.0027 .$. . In Fig. 15, the beginning of such a trajectory is represented. It reached perfect synchronization after 11382 iterations. After the initial period to reach approximate synchronization, there were only very small desynchronization bursts.

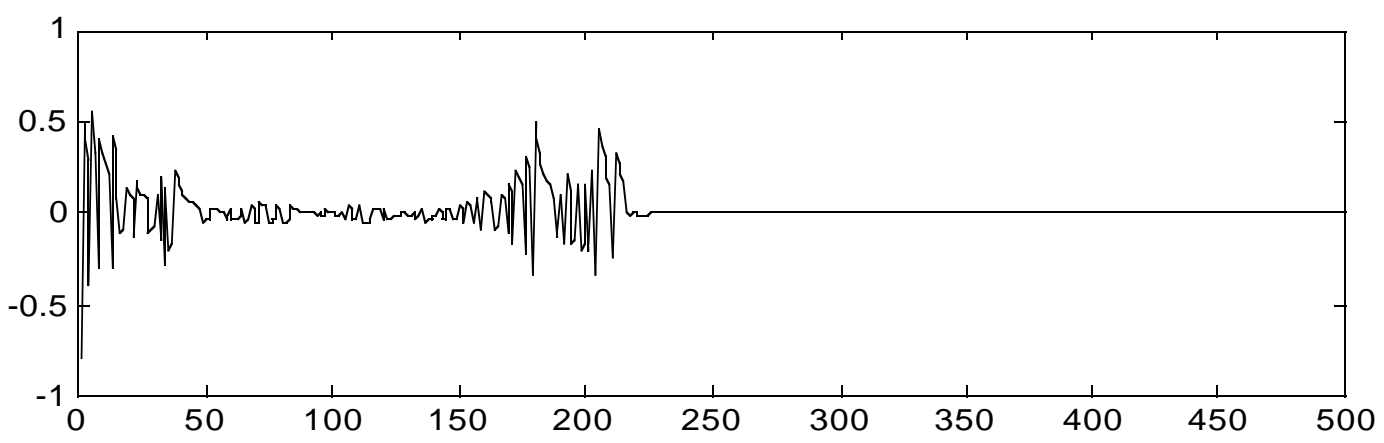

Fig. 15. Synchronization error of a trajectory of two skew tent maps coupled according to version 2 in master-slave configuration, with $\delta=0$ and $\varepsilon=0.484$, starting from $\mathrm{x}(1)=0.9, \mathrm{y}(1)=0.1$.

From these simulations, it appears that the version 1 and version 2 coupled systems with a negative natural transversal Lyapunov exponent, but some trajectories with a positive transversal Lyapunov exponent have a similar qualitative synchronization behavior. This radically changes, as soon as some non-idealities are introduced.

Of course, when in the version 2 coupled system the transversal Lyapunov exponents of all trajectories are negative, the qualitative behavior is different. It is well characterized by inequality (5.8).

\section{The effect of noise and parameter mismatch}

In information transmission applications, where the coupled tent maps belong to the transmitter and the receiver, respectively, the transmitted signal will be corrupted by noise. This can be modeled by the following equations for the master-slave configuration $(\delta=0)$

\section{Version 1:}

$$
\left(\begin{array}{l}
x(k+1) \\
y(k+1)
\end{array}\right)=F\left(\begin{array}{l}
x(k) \\
y(k)
\end{array}\right)=\left(\begin{array}{c}
f[x(k)] \\
f[y(k)]+\varepsilon(x(k)-y(k))+z(k)
\end{array}\right) \quad k=0,1,2, \ldots
$$

\section{Version 2:}




$$
\left(\begin{array}{l}
x(k+1) \\
y(k+1)
\end{array}\right)=\mathbf{G}\left(\begin{array}{l}
x(k) \\
y(k)
\end{array}\right)=\left(\begin{array}{c}
f[x(k)] \\
f[y(k)+\varepsilon(x(k)-y(k))+z(k)]
\end{array}\right) \quad k=0,1,2, \ldots
$$

where $\mathrm{z}(\mathrm{k})$ are identically distributed independent gaussian random variables.

The synchronization error of a solution of (6.1) with standard deviation 0.001 of the gaussian noise $\mathrm{z}(\mathrm{k})$ and $\varepsilon=1.229$, which corresponds to a natural transversal Lyapunov exponent of -0.1401 .. is represented in Fig. 16.

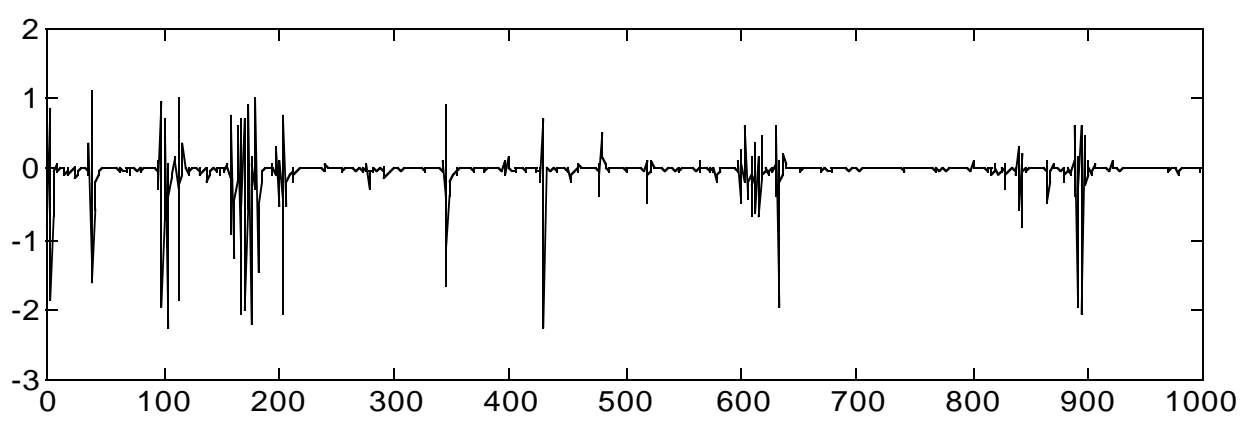

Fig.16. Synchronization error of a trajectory of two version 1 master-slave coupled tent maps with parameter $\mathrm{a}=0.63$ and coupling constant $\varepsilon=1.229$ (natural transvesal Lyapunov exponent -0.1401..) with additive gaussion noise (cf. eq. (6.1)) of standard deviation 0.001 .

The noise term $\mathrm{z}(\mathrm{k})$ renders the convergence to $\mathrm{S}$ impossible and amplifies the effect of the transversally repulsive trajectories of $\mathrm{S}$ to large intermittent desynchronization bursts.

Whe we use version 2 coupling with gaussian noise, according to equation (6.2) with e = 0.55, which corresponds to a natural transversal Lyapunov exponent of $-0.1396 .$. , about the same as for the version 1 coupled trajectory, the behavior is quite different. Again, perfect synchronization is made impossible by the noise term, but the solution, after an initial time interval needed to reach approximate synchronization, remains approximately synchronized and the synchronization error has the aspect of small noise (Fig.17) rather than intermittent large bursts as in Fig.16. Note that the scale of the vertical axis differs by two orders of magnitude in Fig. 16 and Fig.17.

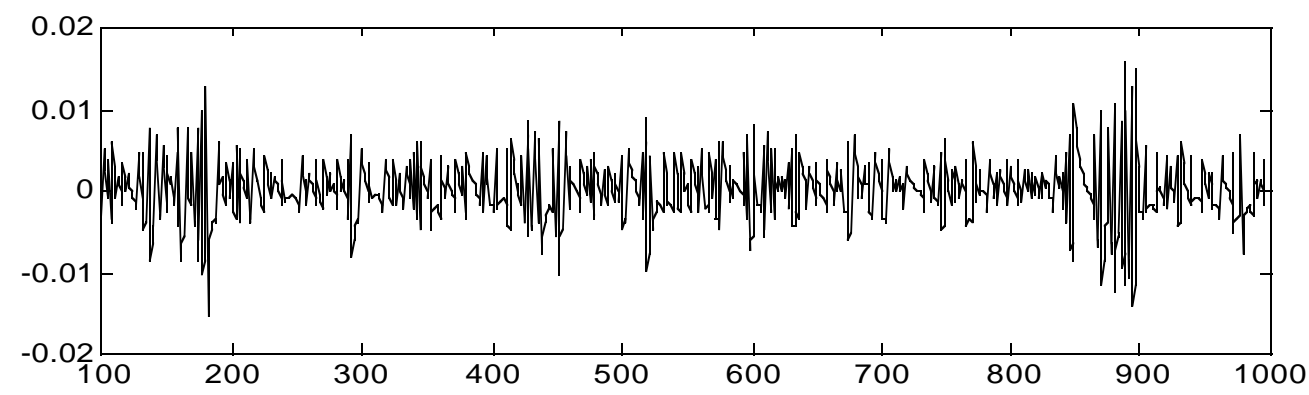


Fig.17. Synchronization error of a trajectory of two version 2 master-slave coupled tent maps with parameter $\mathrm{a}=0.63$ and coupling constant $\varepsilon=0.55$ (natural transversal Lyapunov exponent -0.1396..) with additive gaussion noise (cf. eq. (6.2)) of standard deviation 0.001 .

For the coupling constant $\varepsilon=0.55$ there are still trajectories in $S$ with positive transversal Lyapunov exponents, whereas for $\varepsilon$ in the interval $(0.4826 . ., 1.5174 .$.$) all trajectories in S$ have a negative Lyapunov exponent. In this case, one can give an explicit bound on the standard deviation of the synchronization error in terms of the standard devation of the noise term.

\section{Theorem 6.1}

Suppose that in equation (6.2) the random variables $\mathrm{z}(\mathrm{k})$ are independent, equally distributed and gaussian with mean 0 and variance $\sigma^{2}$. Then if

$$
1-\Delta_{0}<\varepsilon<1+\Delta_{0}, \text { where } \Delta_{0}=\min \{\mathrm{a}, 1-\mathrm{a}\}
$$

the variance of any solution of (6.2) starting in $[0,1]^{2}$ is bounded by

$$
\mathrm{E}(\mathrm{x}(\mathrm{k})-\mathrm{y}(\mathrm{k}))^{2}<\frac{\sigma^{2}}{1-\gamma^{2}}+\eta
$$

for any $\eta>0$ and sufficiently large $k$, where

$$
\gamma=\max \left\{\frac{|1-\varepsilon|}{\mathrm{a}}, \frac{|1-\varepsilon|}{1-\mathrm{a}}\right\}
$$

\section{Proof:}

The synchronization error satisfies

$$
\mathrm{x}(\mathrm{k}+1)-\mathrm{y}(\mathrm{k}+1)=\mathrm{f}[\mathrm{x}(\mathrm{k})]-\mathrm{f}[(1-\varepsilon) \mathrm{y}(\mathrm{k})+\varepsilon \mathrm{x}(\mathrm{k})]-\mathrm{z}(\mathrm{k})
$$

Since $z(k)$ is independent of $x(k)$ and $y(k)$, the variances satisfy

$$
\begin{aligned}
\mathrm{E}(\mathrm{x}(\mathrm{k}+1)-\mathrm{y}(\mathrm{k}+1))^{2} & =\mathrm{E}(\mathrm{f}[\mathrm{x}(\mathrm{k})]-\mathrm{f}[(1-\varepsilon) \mathrm{y}(\mathrm{k})+\varepsilon \mathrm{x}(\mathrm{k})])^{2}+\sigma^{2} \\
& \leq \mathrm{E}\left(\max \left(\frac{1}{\mathrm{a}}, \frac{1}{1-\mathrm{a}}\right)|1-\varepsilon| \mid \mathrm{x}(\mathrm{k})-\mathrm{y}(\mathrm{k})\right)^{2}+\sigma^{2} \\
& =\gamma^{2} \mathrm{E}(\mathrm{x}(\mathrm{k})-\mathrm{y}(\mathrm{k}))^{2}+\sigma^{2}
\end{aligned}
$$

Hence, as long as for some $\eta>0$ inequality (6.4) is not satisfied, i.e. as long as

$$
\sigma^{2} \leq\left(1-\gamma^{2}\right) \mathrm{E}(\mathrm{x}(\mathrm{k})-\mathrm{y}(\mathrm{k}))^{2}-\left(1-\gamma^{2}\right) \eta
$$

we get 


$$
\mathrm{E}(\mathrm{x}(\mathrm{k}+1)-\mathrm{y}(\mathrm{k}+1))^{2} \leq \mathrm{E}(\mathrm{x}(\mathrm{k})-\mathrm{y}(\mathrm{k}))^{2}-\left(1-\gamma^{2}\right) \eta
$$

which implies that (6.4) must be satisfied after a finite number of iterations.

Let us check the bound (6.4) by simulation. For $\mathrm{a}=0.63$ and $\varepsilon=0.88$, the natural transversal Lyapunov exponent is -1.4613 .. . The constant $\gamma$ is 0.3243 .. and for $\sigma=0.001$ we get for the asymptotic bound for the standard deviation of the synchronization error

$$
\frac{\sigma}{\sqrt{1-\gamma^{2}}}=0.0011 \text {. }
$$

The synchronization error of a solution of (6.2) with these parameters is represented in Fig.18. The standard deviation estimated from this solution is 0.0010 . which indeed is smaller than (6.10), but very close to it. We represented the solution and estimated the standard deviation only after 100 iterations, in order to avoid initial transient effects.

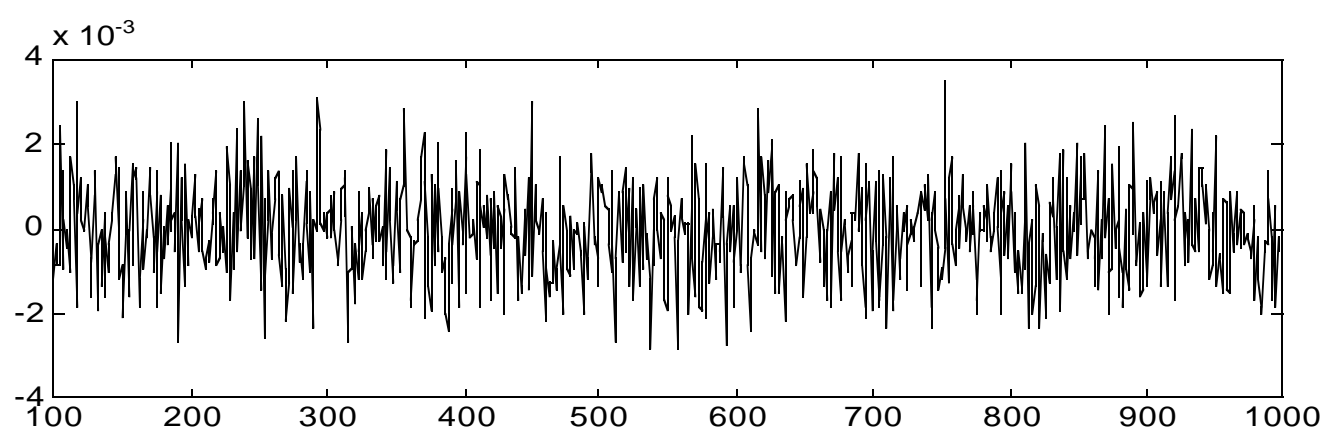

Fig.18. Synchronization error of a trajectory of two version 2 master-slave coupled tent maps with parameter $\mathrm{a}=0.63$ and coupling constant $\varepsilon=0.88$ (natural transversal Lyapunov exponent -1.4613..) with additive gaussion noise (cf. eq. (6.2)) of standard deviation 0.001 .

To compare the behavior of this solution of (6.2) with a solution of the version 1 coupled system (6.1) we chose $\varepsilon=1.545$, which gives a relatively large negative Lyapunov exponent: $1.4575 .$. , very close to the value for the solution of Fig.18. The synchronization error is represented in Fig.19. Despite the relatively large negative natural transversal Lyapunov exponent, large desynchronization bursts are still present. They are three orders of magnitude larger than the synchronization error in Fig.18. With respect to the version 1 coupled maps with a 10 times less negative natural transversal Lyapunov exponent (Fig.16), the large desynchronization bursts are of the same order of magnitude, but simply less frequent. 


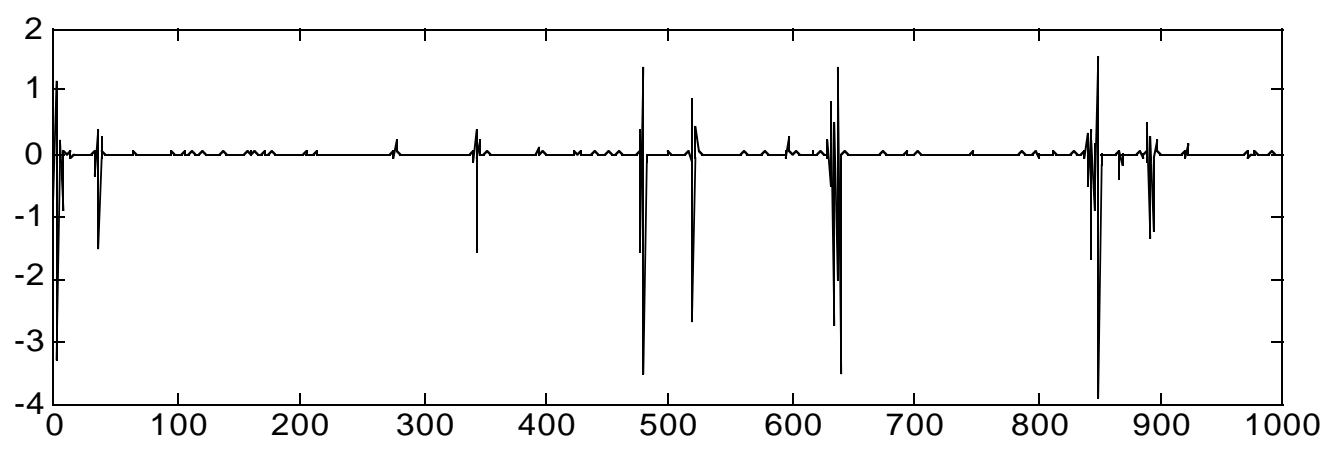

Fig.19. Synchronization error of a trajectory of two version 1 master-slave coupled tent maps with parameter $\mathrm{a}=0.63$ and coupling constant $\varepsilon=1.545$ (natural transversal Lyapunov exponent -1.4575..) with additive gaussion noise (cf. eq. (6.1)) of standard deviation 0.001 .

Another effect that has to be taken into account in practical applications is the mismatch between the parameters of the master and the slave system. In our case, the parameter a of the master and the slave system is different. The equations (4.1) and (4.2) become:

\section{Version 1:}

$$
\left(\begin{array}{l}
x(k+1) \\
y(k+1)
\end{array}\right)=F\left(\begin{array}{l}
x(k) \\
y(k)
\end{array}\right)=\left(\begin{array}{l}
f[x(k)]+\delta(y(k)-x(k)) \\
g[y(k)]+\varepsilon(x(k)-y(k))
\end{array}\right) \quad k=0,1,2, \ldots
$$

\section{Version 2:}

$$
\left(\begin{array}{l}
x(k+1) \\
y(k+1)
\end{array}\right)=G\left(\begin{array}{l}
x(k) \\
y(k)
\end{array}\right)=\left(\begin{array}{l}
f[x(k)+\delta(y(k)-x(k))] \\
g[y(k)+\varepsilon(x(k)-y(k))]
\end{array}\right) \quad k=0,1,2, \ldots
$$

where $\mathrm{f}$ is given by (2.1) and $\mathrm{g}$ by the same equation, but with the parameter $\mathrm{a}+\Delta \mathrm{a}$ instead of a. In this case, the subspace $S$ is not invariant anymore.

Again, we can bound the synchronization error for the version 2 coupled system, when all trajectories of the system without parameter mismatch synchronizes.

\section{Theorem 6.2}

Suppose that in equation (6.12) the coupling parameters satisfy

$$
1-\Delta_{0}<\mathrm{d}<1+\Delta_{0} \text {, where } \Delta_{0}=\min \{\mathrm{a}, 1-\mathrm{a}\} \text { and } \mathrm{d}=\delta+\varepsilon
$$

The the synchronization error of any solution of (6.12) starting in $[0,1]^{2}$ is bounded by

$$
|\mathrm{x}(\mathrm{k})-\mathrm{y}(\mathrm{k})|<\frac{\Delta \mathrm{f}}{1-\gamma}+\eta
$$

for any $\eta>0$ and sufficiently large $\mathrm{k}$, where 


$$
\gamma=\max \left\{\frac{|1-\mathrm{d}|}{\mathrm{a}}, \frac{|1-\mathrm{d}|}{1-\mathrm{a}}\right\}
$$

and

$$
\Delta \mathrm{f}=\max \left\{\frac{|\Delta \mathrm{a}|}{\mathrm{a}}, \frac{|\Delta \mathrm{a}|}{1-\mathrm{a}}\right\}
$$

\section{Proof:}

The synchronization error satisfies

$$
\begin{aligned}
|\mathrm{x}(\mathrm{k}+1)-\mathrm{y}(\mathrm{k}+1)| \leq & |\mathrm{f}[(1-\delta) \mathrm{x}(\mathrm{k})+\delta \mathrm{y}(\mathrm{k})]-\mathrm{f}[(1-\varepsilon) \mathrm{y}(\mathrm{k})+\varepsilon \mathrm{x}(\mathrm{k})]| \\
& +|\mathrm{f}[(1-\varepsilon) \mathrm{y}(\mathrm{k})+\varepsilon \mathrm{x}(\mathrm{k})]-\mathrm{g}[(1-\varepsilon) \mathrm{y}(\mathrm{k})+\varepsilon \mathrm{x}(\mathrm{k})]| \\
\leq & \gamma|\mathrm{x}(\mathrm{k})-\mathrm{y}(\mathrm{k})|+\Delta \mathrm{f}
\end{aligned}
$$

where the maximal difference $\Delta \mathrm{f}$ between the values of $\mathrm{f}$ and $\mathrm{g}$ in [0,1] satisfies (Fig.20)

$$
\Delta \mathrm{f} \leq \max _{\mathrm{x} \in[0,1]}|\mathrm{f}(\mathrm{x})-\mathrm{g}(\mathrm{x})| \leq \max \{|\mathrm{f}(\mathrm{a})-\mathrm{g}(\mathrm{a})|,|\mathrm{f}(\mathrm{a}+\Delta \mathrm{a})-\mathrm{g}(\mathrm{a}+\Delta \mathrm{a})|\}
$$

which yields the bound (6.16).

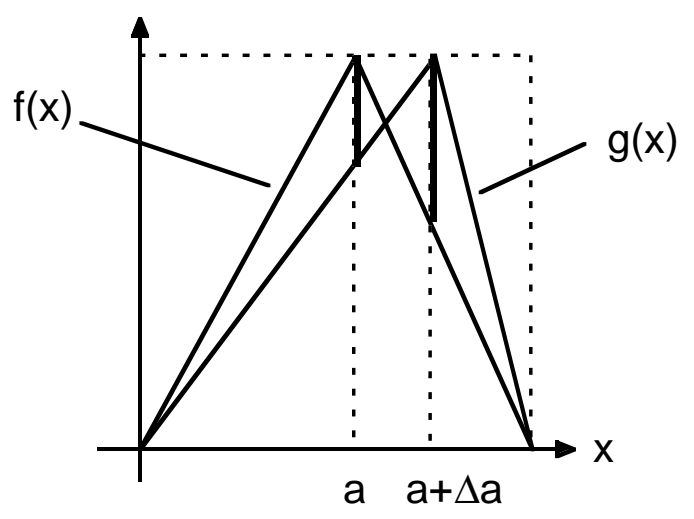

Fig.20. Maximal difference between $\mathrm{f}$ and $\mathrm{g}$

Thus, e.g., a $1 \%$ deviation from $\mathrm{a}=0.63$ for the master-slave coupling $\delta=0, \varepsilon=0.88$ gives an asymptotic bound of 0.0252 for the synchronization error, according to (6.14). The results of a numerical simulation is shown in Fig.21. It confirms the validity of the bound. 


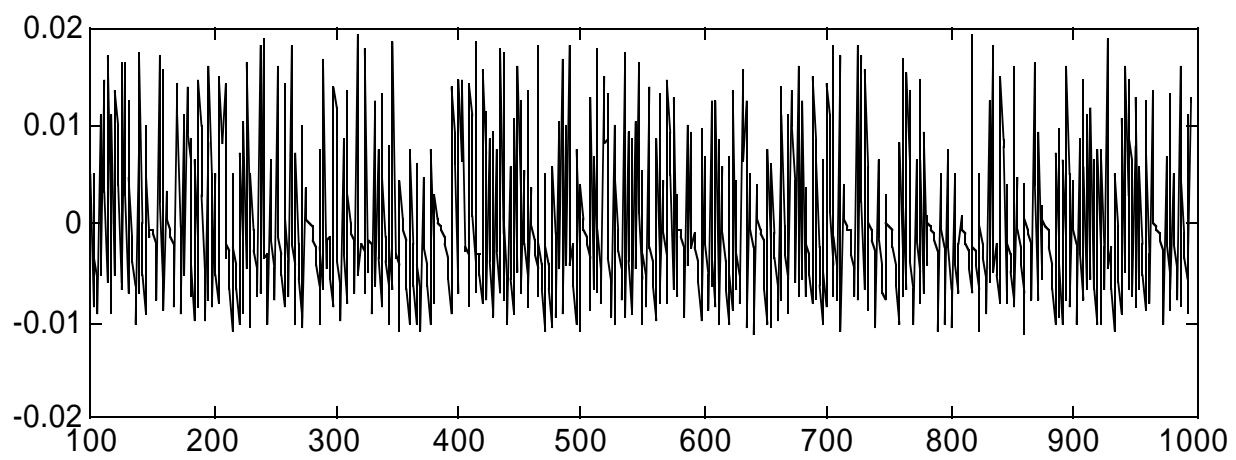

Fig.20. Synchronization error of a trajectory of two version 2 master-slave coupled tent maps with parameters $\mathrm{a}=0.63$ and $\mathrm{a}+\Delta \mathrm{a}=0.6363$ and coupling constant $\varepsilon=0.88$ (natural transversal Lyapunov exponent -1.4613..).

For comparison, again, the synchronization error of the same maps, but coupled according to version 1 such that the system has approximately the same natural transversal Lyapunov exponent, is represented in Fig.21. Again, the version 1 coupled maps have large desynchronization bursts.

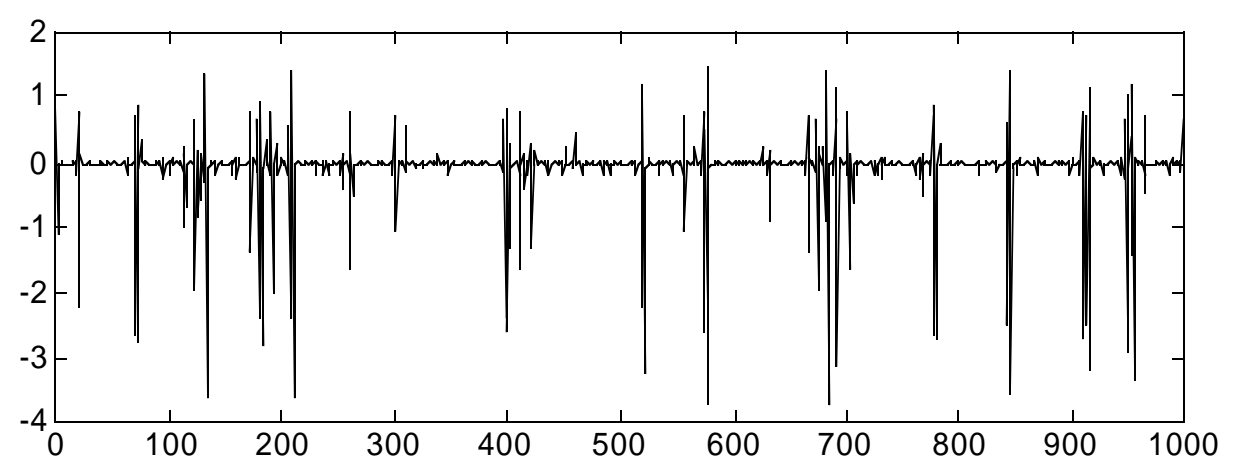

Fig.21. Synchronization error of a trajectory of two version 1 master-slave coupled tent maps with parameters $\mathrm{a}=0.63$ and $\mathrm{a}+\Delta \mathrm{a}=0.6363$ and coupling constant $\varepsilon=$ 1.545 (natural transversal Lyapunov exponent $-1.4575 .$.$) .$

\section{Conclusion}

The synchronization behavior of two either linearly of nonlinearly coupled skew tent maps has been discussed using the transversal Lyapunov exponent of the synchronized trajectories.

When the transversal Lyapunov exponent of all synchronized trajectories is negative, all trajectories synchronize and when small parameter mismatch occurs or noise is present in the system, the synchronization error becomes asymptotically small .

When the natural transversal Lyapunov exponent is negative, but some trajectories still have a positive Lyapunov exponent, which is at best the case for linear coupling the basin of 
attraction of the synchronization subspace is locally riddled. The global behavior of the dynamics depends, however, on the particular form of the system. In the case of linear coupling, large desynchronization bursts take place intermittetly.

\section{Acknowledgement:}

This work has been financially supported by the Swiss National Science Foundation, grant.7UKPJ 048229 (cooperation with the CEEC/NIS states, financed by the ministry of foreign affairs) and grant 2000-047172.96.

\section{References:}

[1] H.Fujisaka, T.Yamada, "Stability theory of synchronized motion in coupled oscillator systems", Progr. Theor. Phys., vol. 69, p.32, 1983

[2] A.S.Pikovsky, "On the interaction of strange attractors", Z.Physik, vol. B55, p.149, 1984

[3] V.Afraimovich, N.Veritchev, M.Rabinovich, "Stochastically synchronized oscillators in dissipative systems", Radiophysics and Quantum Electronics, vol.29, p.795, 1986 (in russian)

[4] L.M.Pecora, T.L.Carroll, "Synchronization in Chaotic Systems", Phys. Rev. Lett., vol.64, pp.821-824, 1990.

[5] M.Hasler, "Engineering chaos for encryption and broadband communication", Philosophical Transactions of the Royal Society of London, Trransaction A, vol.353, pp.115-126, 1995.

[6] M.Hasler, "Synchronization of chaotic systems and transmission of information", to appear in Int. J. on Bif. and Chaos.

[7] J.C.Alexander, J.A.Yorke, Z.You, "Riddled basins", Int. J. of Bif. and Chaos, vol. 2, pp.795-813, 1992.

[8] P.Ashwin, J.Buescu, I.Stewart, "Bubbling of attractors and synchronization of chaotic oscillators", Phys. Lett. A, vol. 193, pp.126-139, 1994.

[9] E.Ott, J.C.Sommerer, "Blowout bifurcations: the occurrence of riddled basins", Phys. Lett.A, vol. 188, pp.39-47, 1994.

[10] Y-C.Lai, C.Grebogi, J.A.Yorke, "Riddling bifurcation in chaotic dynamical systems", Phys. Rev. Lett, vol. 77, 1996, pp.55-58, 1996.

[11] P.Ashwin, J.Buescu, I.Stewart, "From attractor to chaotic saddle: a tale of transverse instability", Nonlinearity, vol.9, pp.703-737, 1996.

[12] Y.Maistrenko, T.Kapitaniak, "Different types of chaos synchronization in two coupled piecewise linear maps", Phys. Rev.E., vol.54, pp.3285-3292, 1996. 
[13] A.Dmitriev, M.Shirokov, S.Starkov, "Chaotic synchronization in ensembles of locally and globally coupled discrete-time dynamical systems. Rigorous results and computer simulation". Proceedings of NDES'95, pp.287-290, Dublin, 1995.

[14] J.-P.Eckmann, D.Ruelle, "Ergodic theory of chaos and strange attractors", Rev. Mod. Phys., vol.57, pp.617-656, 1985.

[15] P.Billingsley, Ergodic theory and information, John Wiley, New York, 1965.

[16] V.I.Oseledec, "A multiplicative ergodic theorem. Lyapunov characteristic numbers for dynamical systems", Trans. Moscow Math.Soc., vol.19, pp.197-221, 1968.

[17] Ch.Mira, L.Gardini, A.Barugola, J.-C.Catala, Chaotic dynamics in 2-dimensional noninvertible maps, World Scientific, Singapore, 1996. 